\title{
TOXICIDADE AGUDA DO AGROTÓXICO FIPRONIL AO COPEPODA CYCLOPOIDA MESOCYCLOPS MERIDIANUS - TESTES PRELIMINARES
}

\author{
Renata Martins dos Santos ${ }^{1}$
}

Raquel Aparecida Moreira

Odete Rocha ${ }^{3}$

RESUMO: Embora os Copepoda de água doce geralmente possuam estreitas faixas de tolerância aos fatores ambientais e sejam particularmente sensíveis aos contaminantes contidos na coluna d'água, estes são raramente utilizados como organismos-teste em estudos ecotoxicológicos. O objetivo deste trabalho foi avaliar a toxicidade aguda do agrotóxico fipronil utilizando como organismo-teste a espécie de Copepoda de água doce, Mesocyclops meridianus. Os organismos foram coletados na Represa do Lobo (Broa), São Paulo, SP. Para o teste, a solução controle e as soluções-teste $\left(0,156 \mathrm{mg} \cdot \mathrm{L}^{-1}, 0,312\right.$ $\mathrm{mg} \cdot \mathrm{L}^{-1}, 0,625 \mathrm{mg} \cdot \mathrm{L}^{-1}$ e $\left.1,25 \mathrm{mg} \cdot \mathrm{L}^{-1}\right)$ foram realizadas com quatro repetições para cada uma. Foram colocados cinco indivíduos de $M$. meridianus em cada cavidade da placa de acrílico. Os recipientes-teste foram mantidos em incubadora a $24 \pm 1{ }^{\circ} \mathrm{C}$ com fotoperíodo

\footnotetext{
${ }^{1}$ Bióloga, Programa de Pós-Graduação em Ecologia e Recursos Naturais, Universidade Federal de São Carlos. email: renata cordis@yahoo.com.br

${ }^{2}$ Bióloga, Programa de Pós-Graduação em Ecologia e Recursos Naturais, Universidade Federal de São rlos. email: raquel.moreira88@hotmail.com

${ }^{3}$ Docente do Departamento de Ecologia e Biologia Evolutiva, Universidade Federal de São Carlos. email: doro@ufscar.br
}

VIII Fórum Ambiental da Alta Paulista, v. 8, n. 12, 2012, p. 147-158. 
de 16 horas luz : 8 horas escuro durante o período de 48 h de exposição ao agrotóxico. A mortalidade observada após este período foi utilizada para a determinação da $\mathrm{CL}_{50}$. Para tal cálculo, utilizou-se o programa estatístico "Trimmed Spearman-Karber Method for Estimating Median Lethal Concentrations in Toxicity Biossays". O fipronil foi considerado altamente tóxico para $M$. meridianus durante a exposição de $48 \mathrm{~h}$ e o valor da $\mathrm{CL}_{50}-48 \mathrm{~h}$ foi de $0,99 \mathrm{mg} \mathrm{L}^{-1}$. Analisando-se os resultados deste e de outros estudos observou-se que as espécies de Copepoda de água doce apresentam grande sensibilidade a diferentes agentes tóxicos. A espécie Mesocyclops meridianus apresentou alta sensibilidade ao agrotóxico fipronil e por ser de fácil manipulação laboratorial pode ser um organismo-teste em estudos futuros de ecotoxicologia.

Palavras-chave: Agrotóxico. Copepoda Cyclopoida, fipronil.

\section{INTRODUÇÃO}

Apesar dos Copepoda de água doce possuírem estreitas faixas de tolerância aos fatores ambientais (MATSUMURA-TUNDISI; TUNDISI, 2003) e serem particularmente sensíveis aos contaminantes contidos na coluna d'água (OKUMURA, 2011), estes são raramente utilizados como organismos-teste em estudos ecotoxicológicos. Os copépodos são animais importantes em ecossistemas aquáticos e nestes são encontrados em quase todos os tipos de habitats (REID, 2001). Eles constituem uma importante fonte de alimento para os peixes que são predadores do plâncton, e atuam como um elo na transferência de energia através da cadeia alimentar (RAISUDDIN et al., 2007). Boxshall; Defaye (2008) relataram, em seu estudo sobre a diversidade global de Copepoda, que existem 13.000 espécies de Copepoda conhecidas em todo o mundo com um grande número ainda não descrito, sendo que destas apenas 2.814 habitam os ambientes de água doce.

Calanoida, Cyclopoida e Harpacticoida são as três Ordens de copépodos de vida livre, sendo que os Cyclopoida contêm a família Cyclopidae que possui o maior número de espécies em água doce (BOXSHALL; DEFAYE 2008). Os copépodos Cyclopoida, 
juntamente com rotíferos, são os primeiros a colonizar tanques experimentais (CÁCERES; SOLUK, 2002; COHEN; SHURIN 2003). Os copépodos também desempenham um importante papel como um elo na transferência de energia através dos diferentes níveis da cadeia trófica e são abundantes na maioria dos ecossistemas aquáticos (LAMPERT; SOMMER, 1997; KULKARNI et al., 2012).

Um grande número de estudos para a avaliação dos efeitos tóxicos de uma variedade de contaminantes utiliza os Copepoda marinhos planctônicos como organismos-teste (OKUMURA, 2011). No entanto, os Copepoda de água doce são raramente utilizados em estudos ecotoxicológicos (KULKARNI et al., 2012), apesar de na última década este número ter aumentado. Esses últimos autores, em um estudo de revisão, concluíram que os copépodos de água doce são considerados sensíveis a substâncias tóxicas sendo tão vulneráveis à exposição de contaminantes, se não mais, do que outros organismos de invertebrados tradicionalmente utilizados em Ecotoxicologia Aquática.

Entre os estudos com Copepoda, as espécies da Ordem Calanoida são mais utilizadas em avaliações laboratoriais de toxicidade. No Brasil Gusmão (2003), em estudos em mesocosmos, verificou o efeito tóxico dos metais cobre e cromo sobre populações naturais de Cladocera, Copepoda e Rotifera. Entre as espécies mais sensíveis a ambos os metais, se destacaram Argyrodiaptomus furcatus e Notodiaptomus iheringi. Os efeitos das concentrações de campo dos herbicidas Clomazone, Quinclorac, Metsulfuron-methyl e Propanil, em lavoura arrozeira, sobre a comunidade zooplanctônica foram determinados em um estudo realizado em viveiros de aqüicultura (REIMCHE et al., 2008). Os resultados indicaram que os herbicidas provocaram poucas alterações na densidade de organismos dos grupos Rotifera e Copepoda.

Ainda não há um protocolo para testes de toxicidade aguda ou crônica com copépodos de água doce (KULKARNI et al., 2012), sendo que a maioria de trabalhos são realizados em mesocosmos com avaliação da comunidade zooplanctônica apenas em nível de grandes grupos. Estudos de microcosmos e mesocosmos realizados para analisar as respostas de comunidades água doce a diferentes toxinas revelaram que os copépodos são potencialmente sensíveis (VAN WIJNGAARDEN et al., 1996; 2006; WENDT-RASCH et al. 2003; VAN DEN BRINK et al. 2009; ZAFAR et al. 2012). Nesses 


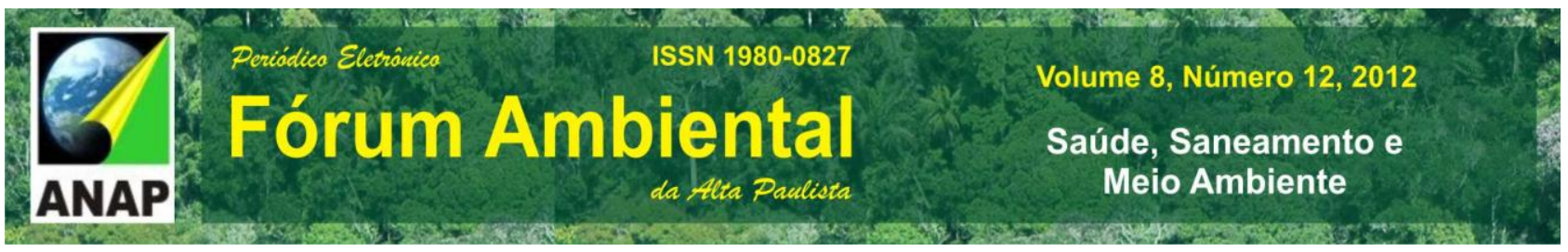

estudos verificou-se que dentre os organismos zooplanctônicos Copepoda foi o grupo mais sensível.

Estudos em nível de espécie foram realizados em testes de toxicidade aguda e crônica, principalmente em espécies marinhas de Copepoda Calanoida e Harpacticoida analisando os efeitos de vários metais, óleos, nano-partículas, sedimentos orgânicos e várias misturas (KUSK 1997; MACKEN et al., 2009; HANSEN et al., 2011 apud KULKARNI et al., 2012). Entre os trabalhos revisados por Kulkarni et al. (2012) apenas seis estudos foram realizados com copépodos Cyclopoida, principalmente em estudos de mesocosmos, dos quais apenas dois foram estudos laboratoriais e dois em nível de espécie.

O zooplâncton pode ser afetado desde o nível individual até de comunidade por muitas substâncias químicas tóxicas. Dentre estas, os agrotóxicos são substâncias amplamente utilizadas na agricultura e uma vez nos corpos d'água se tornam uma fonte de contaminação que se espalham por todo o sistema, sendo difícil conter sua dispersão e ação tóxica sobre outros organismos (GOODRICH; LEACH, 1990).

Os agrotóxicos são um dos produtos mais amplamente encontrados em corpos hídricos superficiais e subterrâneos em todo o mundo devido ao seu amplo uso em áreas agrícolas e urbanas (CELLA, 2009). Nos ecossistemas aquáticos estes elementos podem permanecer dissolvidos na coluna d'água, alcançar o sedimento ou se acumularem nos tecidos dos organismos (PERET, 2009).

De todo o agrotóxico aplicado nas lavouras agrícolas, em média, apenas $0,1 \%$ do produto atinge os organismos-alvo (PIMENTEL; LEVITAM, 1991), o restante pode ser carreado pelas águas das chuvas e contaminar principalmente os ecossistemas aquáticos próximos. Portanto, é no ambiente aquático que grande quantidade destes compostos irá se acumular (RAND, 1995)

Um inseticida utilizado em grandes quantidades em lavouras é o fipronil que é considerado um pesticida seletivo que atua no sistema nervoso paralisando ou matando o organismo alvo (RHOUNE-POULENC, 1996). O fipronil é um composto não-iônico (PIASAROLO, 2008), utilizado como produto de uso caseiro em forma de isca, para o controle de baratas, formigas, grilos e cupins (KIDD; JAMES, 1991). 


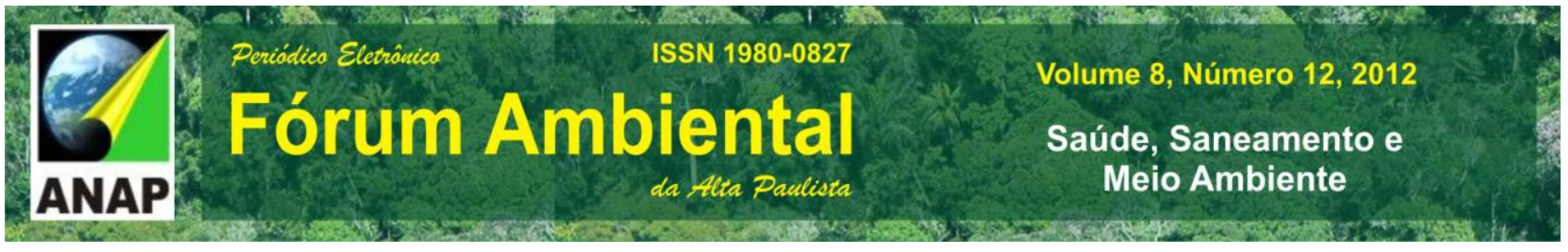

No Brasil, o fipronil é um pesticida utilizado desde o ano 2000 em plantações da região Nordeste. No país seu uso é intenso principalmente em culturas de cana-de-açúcar para controle de formigas, cupins e larvas de coleópteros (HAINZL; CASIDA, 1996). Após sua aplicação, o fipronil pode ser quimicamente degradado ou biodegradado gerando vários subprodutos que podem ser mais nocivos ao meio ambiente e aos organismos ali presentes do que o composto original.

No entanto, para algumas espécies de peixes e moluscos o fipronil é apontado como um pesticida de baixo efeito tóxico já para insetos é altamente seletivo (WIRTH et al., 2004; NGIM; CROSBY, 2001; MESLÉARD et al., 2005; CHATON et al., 2001 apud PERET, 2009). O mesmo não se verifica para os produtos de sua degradação.

Comparando-se os valores da $\mathrm{CL}_{50}$ para os diferentes grupos de organismos já testados em relação a toxicidade do fipronil verifica-se que os valores da concentração letal para os insetos são extremamente baixos, quando se compara com os efeitos em outros organismos aquáticos, como crustáceos, moluscos ou mesmo alguns peixes (GUNASEKARA; TROUNG, 2007).

No desenvolvimento de proteção específica uma das opções de meta para a avaliação de risco ambiental de agrotóxicos, em particular no que diz respeito à revisão dos documentos de orientação em Ecotoxicologia Aquática e Terrestre (EFSA, 2010), é encontrar espécies chave (grupos taxonômicos representativos) e também identificar as espécies vulneráveis dos grupos chave. Estas podem não ser necessariamente as espécies comuns, mas podem ser espécies para as quais a sensibilidade toxicológica, bem como as peculiaridades do ciclo de vida possam influenciar a capacidade de recuperação do ambiente (EFSA 2010).

Portanto, o estudo da sensibilidade de uma gama de organismos pode auxiliar as agências reguladoras e fiscalizadoras na tomada de medidas para manter a integridade dos corpos d'água e com isso proteger a saúde da espécie humana, a preservação da biota aquática e dos serviços ecossistêmicos. Desta forma, o objetivo deste trabalho foi determinar a sensibilidade de Mesocyclops meridianus ao inseticida fipronil. 


\section{MATERIAIS E MÉTODOS}

\section{Origem do material biológico}

Os indivíduos da espécie Mesocyclops meridianus Keifer, 1926 foram coletados na Represa do Lobo $\left(22^{\circ} 15^{\prime} \mathrm{S}\right.$ e $\left.47^{\circ} 49^{\circ} \mathrm{W}\right)$ que está localizada na bacia hidrográfica do Lobo e apresenta uma área de aproximadamente $222 \mathrm{~km}^{2}$, com altitude máxima de $970 \mathrm{~m}$ e mínima de $700 \mathrm{~m}$. Esta represa apresenta $8 \mathrm{~km}$ de comprimento máximo, profundidade máxima de $12 \mathrm{~m}$ e média de $3 \mathrm{~m}$, o tempo de retenção da água varia de 20 a 40 dias (TUNDISI; MATSUMURA-TUNDISI, 1995).

Os indivíduos foram coletados, isolados da amostra e submetidos ao teste. Esse estudo é um trabalho preliminar e posteriormente deverão ser realizados outros testes com os mesmos organismos, porém, já adaptados às condições de cultivo. Desse modo, posteriormente poderão ser comparados os resultados da sensibilidade da espécie Mesocyclops meridianus ao agrotóxico fipronil em relação ao método de adaptação dos indivíduos.

\section{Testes de toxicidade aguda}

Os testes de toxicidade aguda foram realizados utilizando-se o produto comercial Regente 800 WG ${ }^{\circledR}$ (80\% fipronil e $20 \%$ celulose) como fonte de fipronil. Preparou-se a solução estoque em água destilada na concentração de $1 \mathrm{~g} \mathrm{~L}^{-1}$.

Para evitar a degradação do agroquímico nesta solução estoque a mesma foi mantida em frasco totalmente escuro e em freezer comum $\left(-15^{\circ} \mathrm{C}\right)$ e posteriormente foram feitas as diluições desejadas.

A solução controle e as soluções-teste $\left(0,156 \mathrm{mg} \cdot \mathrm{L}^{-1}, 0,312 \mathrm{mg} \cdot \mathrm{L}^{-1}, 0,625 \mathrm{mg} \cdot \mathrm{L}^{-1} \mathrm{e}\right.$ $1,25 \mathrm{mg} \cdot \mathrm{L}^{-1}$ ) com quatro repetições para cada uma das concentrações foram colocadas em placas de cultivo de tecido de poliestireno (Costar®). Em cada cavidade da placa de cultivo foram colocados aleatoriamente cinco organismos da espécie Mesocyclops meridianus, sendo machos e fêmeas (copepoditos IV, V e adultos). Em seguida as placas de cultivo com os organismos foram mantidos em incubadora a $24 \pm 1^{\circ} \mathrm{C}$ com fotoperíodo 
de 16 horas luz/8 horas escuro com o tempo de 24 e 48 horas de exposição ao agrotóxico.

Foram observados nas leituras do teste de toxicidade aguda em 24 horas e 48 horas o número de organismos mortos, sob lupa, somando-se o número total de indivíduos mortos nas réplicas do controle e em cada concentração testada. O resultado relativo à mortalidade observada foi utilizado para a determinação da $\mathrm{CL}_{50}$ (concentração letal para $50 \%$ dos organismos). Para tal cálculo, utilizou-se o programa estatístico "Trimmed Spearman-Karber Method for Estimating Median Lethal Concentrations in Toxicity Biossays" (HAMILTON et al., 1977).

\section{RESULTADOS E DISCUSSÃo}

O valor da $\mathrm{CL}_{50}$ para espécie Mesocyclops meridianus em 48 horas foi de 0,99 mg $\mathrm{L}^{-1}$ de fipronil. As classes de toxicidade dos agrotóxicos em relação as $\mathrm{CL}_{50}$ e $\mathrm{CE}_{50}$ para organismos aquáticos foram compiladas por Zucker (1985) e são apresentas na tabela 1. De acordo com a tabela, o fipronil pode ser considerado altamente tóxico para o copépodo M. meridianus.

Tabela 1 - Classes de toxicidade aguda de agrotóxicos para organismos aquáticos. Fonte: Zucker, 1985.

\begin{tabular}{lc}
\hline Classe de toxicidade & $\mathrm{CL}_{50} \mathbf{O u ~}_{\mathbf{C E}}\left(\mathrm{mg} \mathrm{L}^{-1}\right)$ \\
\hline Extremamente tóxico & $<0,1$ \\
Altamente toxico & $0,1 \mathrm{a} 1,0$ \\
Moderadamente tóxico & $>1,0 \mathrm{a}<10$ \\
Ligeiramente tóxico & $>10 \mathrm{a}<100$ \\
Praticamente não tóxico & $>100$ \\
\hline
\end{tabular}

$\mathrm{Na}$ figura 1 estão representados os valores da mortalidade dos indivíduos de $M$. meridianus expostos ao agrotóxico fipronil durante $48 \mathrm{~h}$. 


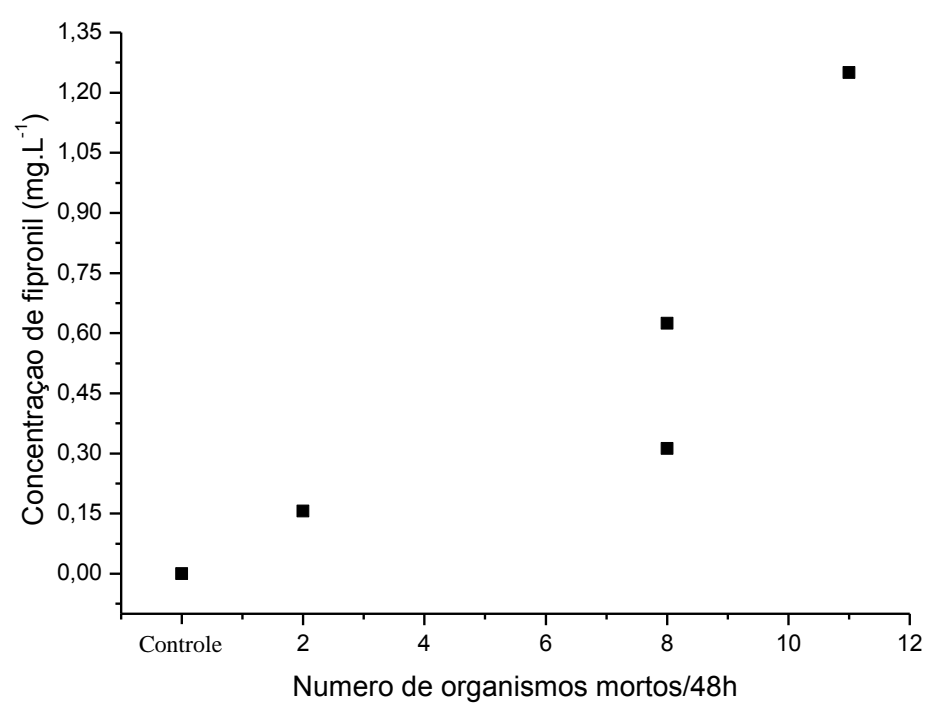

Figura 1. Efeito tóxico de diferentes concentrações de fipronil ao Copepoda Cyclopoida Mesocyclops meridianus após 48h de exposição, confiança de 95\%, teste Trimmed-Spearmann-Karber (TSK).

Os testes ecotoxicológicos com copépodes são frequentemente realizados com sedimentos marinhos, metais tóxicos como cobre, seguidos por componentes de efluentes e pesticidas (KULKARNI et al., 2012). Na literatura até o momento, não foi descrito teste da sensibilidade da espécie Mesocyclops meridianus ao agrotóxico fipronil. No entanto este produto já foi testado para outras espécies de peixes, moluscos, cladóceros e larva de insetos. Dentre estes, o organismo mais sensível foi de Chironomus crassicaudatus $\left(0,48 \mu \mathrm{g} \cdot \mathrm{L}^{-1}\right)$.

Mesocyclops meridianus é uma espécie de ampla distribuição e registrou elevada sensibilidade ao fipronil podendo desta forma ser futuramente um organismo-teste padronizado. Kulkarni et al. (2012), após ampla revisão, concluíram que a espécie $M$. leuckarti, por possuir um ciclo de vida relativamente curto, ser uma espécie de verão e por ser uma das espécies mais bem estudadas de Cyclopoida de água doce na Europa e em particular na Alemanha pode ser selecionada como uma espécie de Copepoda de água doce para os testes ecotoxicológicos. Outros trabalhos também reportam a facilidade de manutenção em cultivo de espécies do gênero Mesocyclops (LEBOULANGER et al., 2011).

Estes autores verificaram que os náuplios de $M$. aspericornis são sensíveis tanto aos herbicidas Diuron e Paraquat, quanto aos inseticidas Temphos e Fenitrothion e 
concluíram que é necessário o isolamento de organismos modelos em ecossistemas tropicais para cumprir as exigências para o padrão de avaliação dos riscos ambientais.

\section{CONCLUSÕES}

Recentemente verifica-se um aumento na quantidade de estudos que abordam os aspectos biológicos e ecotoxicológicos dos copépodos de água doce. O fato da espécie Mesocyclops meridianus possuir uma elevada sensibilidade ao agrotóxico fipronil e alta taxa reprodutiva e, também por ser de fácil manipulação laboratorial sugere que espécies deste grupo poderão ser organismos-teste adequados para estudos dos riscos ambientais.

A sensibilidade da espécie de Copepoda Mesocyclops meridianus evidencia que estes microcrustáceos são vulneráveis à contaminação por fipronil e que os corpos de água próximos às culturas onde este pesticida é aplicado precisam ser protegidos e manejados para prevenir as perdas de diversidade da biota de água.

\section{REFERÊNCIAS BIBLIOGRÁFICAS}

BOXSHALL, G. A.; DEFAYE, D. Global diversity of copepods (Crustacea: Copepoda) in freshwater. Hydrobiologia,v. 595, p. 195-207, 2008.

CACERES, C. E.; SOLUK, D. A. Blowing in the wind: a field test of overland dispersal and colonization by aquatic invertebrates. Oecologia, v. 131, p. 402-408, 2002.

CELLA, A. L. Ecologia do agrotóxico fipronil em pacu (Piaractus mesopotamicus Holmerg) e paulistinha (Danio rerio) e resíduos de agrotóxicos na bacia do rio Corumbataí. 2009. 93 p.Tese (Doutor em Ciências). Universidade de São Paulo, Centro de Energia Nuclear na Agricultura, Piracicaba, 2009

COHEN, G. M.; SHURIN, J. B. Scale-dependence and mechanisms of dispersal in freshwater zooplankton. Oikos 103:603-617, 2003.

EFSA (2010) Panel on Plant Protection Products and their Residues: scientific opinion on the development of specific protection goal options for environmental risk assessment of pesticides, in particular in relation to the revision of the Guidance Documents on Aquatic and Terrestrial Ecotoxicology (SANCO/3268/2001 and SANCO/10329/2002). EFSA J 8:1821. 
GOODRICH, M. S.; LEACH, J. J. A behavioral screening assay for Daphnia magna: a method to assess the effects of xenobiotics on spatial orientation. Environmental Toxicology and Chemistry, Houston, v.9, p.21-30, 1990.

GUNASEKARA, A. S.; TROUNG, T. Environmental Fate of Fipronil Environmental Monitoring Branch California, 2007.

GUSMÃO, L. F. M. Efeitos do cobre e cromo na comunidade zooplanctônica: um estudo experimental em mesocosmos. 2003. 268 p. Tese (Mestrado em Ciências da Engenharia Ambiental), Universidade de São Paulo, Escola de Engenharia de São Carlos, São Carlos, 2003

HAINZL, D., CASIDA, J. E., 1996. Fipronil insecticide: novel photochemical desulfinylation with retention of neurotoxicity. Proc. Natl. Acad. Sci. USA 93, 12764-12767.

HAMILTON, M.A., RUSSO, R.C. \& THURFTON, R.B. Trimmed Spearman-Karber method for estimating median lethal concentration in toxicity bioassays.

Environmental Science \& Technology, 11 (7): 714-719, 1977.

KIDD, H. and D.R. JAMES, Eds. The Agrochemicals Handbook, Third Edition. Royal Society of Chemistry Information Services, Cambridge, UK, 1991, 3-11.

KULKARNI, D.; GERGS, A.; HOMMEN, U.; RATTE, H. T.; PREUSS, T. G. A plea for the use of copepods in freshwater ecotoxicology. Environ Sci Pollut Res., 2012.

LAMPERT, W.; SOMMER, U. Limnoecology: the ecology of lakes and streams. Oxford University Press, New York, 1997.

LEBOUlANGER, C.; SCHWARTZ, C.; SOMVILLE, P.; DIALLO, A. O.; PAGANO, M. Sensitivity of two Mesocyclops (Crustacea, Copepoda, Cyclopidae), from tropical and temperate origins, to the herbicides, diuron and paraquat, and the insecticides, temephos and fenitrothion. Bull Environ Contam Toxicol., v. 87,p. 487-493, 2011.

MATSUMURA-TUNDISI, T.; TUNDISI, J. G. Calanoida (Copepoda) species composition changes in the reservoirs of São Paulo State (Brazil) in the last twenty years.

Hydrobiologia, v. 504, p. 215-222, 2003.

OKUMURA, D. T. Estudos ecotoxicológicos com as espécies Argyrodiaptomus azevedoi e Notodiaptomus iheringi (Copepoda, Calanoida). 2011. 338 p. Tese (Doutorado em Ciências da Engenharia ambiental). Universidade de São Paulo, São Carlos, 2011.

PERET, A. M. Quantificação do pesticida fipronil em um alagoa marginal do Rio Moji-Guaçu e a cinética de sua degradação por microrganismos aquáticos. 2009, 104 p. Tese (Doutorado em Ciências). Universidade Federal de São Carlos, 2009. 
PIASAROLO, L.; DE OLIVEIRA, R.R.L.; GUERREIRO, M.C. Influência da polaridade de pesticidas não-iônicos sobre sua sorção em um latossolo. Ciênc. Agrotec., v.32, n.6 p.1802-1809, 2008.

PIMENTEL, D.; LEVITAM, L. Pesticide: amounts applied and amounts reaching plants. BioScience, v. 36, p. 86-91, 1986.

RAISUDDIN, S.; KWOK, K. W. H; LEUNG, K. M. Y, SCHLENK, D.; LEE, J.S. The copepod Tigriopus: a promising marine model organism for ecotoxicology and environmental genomics. Aquatic Toxicology, v. 83, p. 161-173, 2007.

RAND, G.M. 1995. Fundamentals of Aquatic Toxicology: effects, environmental fate and risk assessment. New York-NY. Taylor e Francis, $1124 \mathrm{p}$.

REID, J. W. A human challenge: discovering and understanding continental copepod habitats. Hydrobiologia, v. 453, n. 454, p. 201-226, 2001.

REIMCHE, G. B.; MACHADO, S. L. O.; GOLOMBIESKI, J. L.; BAUMART, J. S.; BRAUN, N.; MARCHESAN, E.; ZANELLA, R. Persistência na água e influência de herbicidas utilizados na lavoura arrozeira sobre a comunidade zooplanctônica de Cladocera, Copepoda e Rotifera. Ciência Rural, v. 38, n. 1, p. 7-13, 2008.

RHÔNE-POULENC AG COMPANY. 1998. Application for Registration. Volume No. 52062-071. Vol 1. Department of pesticide regulation, California Environmental Protection Agency, Sacramento, CA.

TUNDISI, J.G.; MATSUMURA-TUNDISI, T. The Lobo-Broa ecosystem research. In: TUNDISI, J.G.; BICUDO, C.E.M.; MATSUMURA-TUNDISI, T. (eds). Limnology in Brazil. ABC/SBL:Rio de Janeiro, 1995, p. 219-243.

VAN DEN BRINK, P. J.; CRUM, S. J. H.; GYLSTRA, R.; BRANSEN, F.; CUPPEN, J. G. M.; BROCK, T. C. M. Effects of a herbicide-insecticide mixture in freshwater microcosms: risk assessment and ecological effect chain. Environ Pollut., v. 157, p. 237-249, 2009.

VAN WIJNGAARDEN, R. P. A.; VAN DEN BRINK, P. J.; CRU, S. J. H.; OUDE VOSHAAR, J. H.; BROCK, T. C. M.; LEEUWANGH, C. Effects of the insecticide Dursban ${ }^{\circledR} 4 \mathrm{E}$ (active ingredient chlorpyrifos) in outdoor experimental ditches: I. Comparison of short-term toxicity between the laboratory and the field. Environ Toxicol Chem., v. 15, 1133-1142, 1996.

VAN WIJNGAARDEN, R. P. A.; BROCK, T. C. M; VAN DEN BRINK, P. J.; GYLSTRA, R.; MAUND, S. J. Ecological effects of spring and late summer applications of lambdacyhalothrin on freshwater microcosms. Arch Environ Contam Toxicol., v. 50, p. 220239, 2006. 
WENDT-RASCH, L.; FRIBERG-JENSEN, U.; WOIN, P.; CHRISTOFFERSEN, K. Effects of the pyrethroid insecticide cypermethrin on a freshwater community studied under field conditions. II. Direct and indirect effects on the species composition. Aquat Toxicol., v. 63, p. 373-389, 2003.

ZAFAR, M. I.; BELGERS, J. D.M.; VAN WIJNGAARDEN, R. P.A.; MATSER, A.; VAN DEN BRINK, P. J. Ecological impacts of time-variable exposure regimes to the fungicide azoxystrobin on freshwater communities in outdoor microcosms. Ecotoxicol., v. 21, p. 1024-1038, 2012.

ZUCKER, E. Hazard Evaluation Division - Standard Evaluation Procedure - Acute toxicity test for freshwater fish. 1985. (USEPA Publication 540/9-85-006). 